\title{
First class feature abstractions for product derivation
}

\author{
A.G.J. Jansen, R. Smedinga, J. van Gurp and J. Bosch
}

\begin{abstract}
The authors have observed that large software systems are increasingly defined in terms of the features they implement. Consequently, there is a need to express the commonalities and variability between products of a product family in terms of features. Unfortunately, technology support for the early aspect of a feature is currently limited to the requirements level. There is a need to extend this support to the design and implementation level as well. Existing separation of concerns technologies, such as AOP and SOP, may be of use here. However, features are not first class citizens in these paradigms. To address this and to explore the problems and issues with respect to features and feature composition, the authors have formalised the notion of features in a feature model. The feature model relates features to a component role model. Using our model and a composition algorithm, a number of base components and a number of features may be selected from a software product family and a product derived. As a proof of concept, the authors have experimented extensively with a prototype Java implementation of their approach.
\end{abstract}

\section{Introduction}

Software applications grow larger and larger, are maintained for longer periods of time and need to be updated frequently to evolve with new needs and changing consumer requirements. To cope with this increasing size of software applications a software product family (SPF) [1,2] approach can be used. An SPF is designed for a family of (domain) related applications. It consists of a product-line architecture and a set of reusable components. Specific applications may be derived from the SPF by selecting, enhancing and adapting components. We have observed that, during product derivation, the differences between products are usually defined in terms of features [1, 3-5].

Features are an example of early aspects [6], crosscutting concerns at the requirements and architectural level. Features are used in requirement engineering to define optional or incremental units of change [7]. They have a many-to-many relationship to the individual requirements [1]. Tracing features to the implementing SPF components is complex. In ideal cases, a particular feature implementation is localised to a single module; but in many cases features will cut across multiple components [8]. Consequently, features are an important early aspect to consider, as they try to bridge the gap between the problem domain and solution domain [9].

The product derivation process in an SPF is a timeconsuming and therefore expensive process. The reason for this is that there is a mismatch between the way products are defined (i.e. in terms of features) and the variability offered by the SPF. Requirements changes generally result in changing and/or adding features to the SPF. However, typically features have no first class representation in the SPF implementation. During product derivation, developers

(C) IEE, 2004

IEE Proceedings online no. 20040922

doi: 10.1049/ip-sen:20040922

Paper first received 3rd June 2003 and in revised form 26th April 2004

The authors are with the Department of Mathematics and Computing Science, University of Groningen, PO Box 800, 9700 AV, The Netherlands must make adaptations to the SPF's provided feature set in order to implement product specific features. Consequently, changing the implementation to meet new requirements is potentially expensive because code related to one feature may be spread over multiple software components.

Ideally, new or changed features would be captured in separate pieces of code that can be changed and maintained independently. Thus changes during the product derivation would be limited to those pieces of code. The main topic of this paper is giving features a first class representation in SPFs so that during product derivation, product developers may select features from the SPF and reuse them in their products. There are a number of problems associated with this type of product derivation. A key contribution of this paper is that we identify those problems and demonstrate in our approach how these can be worked around or solved.

We use a top-down approach of analysing the issue of feature based product derivation. Our top-down approach starts with the modelling of concepts such as features and SPFs in terms of sets. With the help of this formal description of the feature model composition problems are identified. Several solutions to these composition problems are presented. One of these solutions is used in a prototype implementation that is also presented in this paper. The three contributions of this paper are:

- a feature model, which relates features of an SPF with component roles

- a classification of feature composition problems and potential solutions to these problems

- a demonstration of how features can be realised at the implementation level. This opens the way to automatically derive a product from an SPF based on a selection of available features.

\section{Features in software product families}

To clarify our approach, an examination of how features and SPFs are related is presented. After this, an informal outline of our approach is presented. The approach involves features, actors and roles. At the end of the Section the approach is exemplified using the example of a video shop renting system. 


\subsection{Software product families (SPFs)}

An SPF consists of a base implementation (e.g. B) and a number of features (e.g. $F_{1} \ldots F_{25}$ ). A product may be derived from the SPF by selecting an arbitrary number of these features and combining these with the base implementation (e.g. $B+F_{9}+F_{18}+\cdots+F_{23}$ ). The base implementation itself can also be seen as a set of (standard) features, i.e. an SPF then becomes, for example, $F_{1}+F_{2}+F_{3}+$ $F_{4}+F_{9}+F_{18}+\cdots+F_{23}$, where some features (e.g. 1 to 4 ) are standard features (in FODA these are called mandatory features [3]) and others are optional features. In this paper, base components model entities, which cannot easily be decomposed into features. Legacy code components and domain components are examples of these base components.

The properties of the composition-operator ' + ' are our primary interest in this paper. Of course, it would be ideal if that operator were associative i.e. $\left[\left(F_{1}+F_{2}\right)+F_{3}\right]=$ $\left[F_{1}+\left(F_{2}+F_{3}\right)\right]$, and commutative, i.e. $F_{1}+F_{2}=F_{2}+F_{1}$. Then, a product developer would be able to arbitrarily combine features. The developer of each feature would not need to worry about interaction with other features. This way it would not make any difference at what point in time and/or development a certain feature is brought into a feature composition. However, in general (as will be argued in the following Section) this operator is neither associative nor commutative, because of feature dependencies: one feature may depend on another feature. This is, for example, the case if one feature cannot operate without another feature.

A further complication factor is that the composition of features might introduce feature interaction [7]: a feature interaction is some way in which one or more features modify or influence another feature in describing the system's behaviour set.

An example of feature interaction can be found in Microsoft Outlook. Outlook, a popular e-mail client for Windows, has two related features: 'work off-line' and 'send immediately'. The work off-line feature enables users to use the e-mail client without having a permanent connection to their mail server. While working off-line, Outlook caches the different actions of the user and executes them when the user switches to on-line mode. On the other hand, if the send immediately feature is enabled, a message is sent immediately when a user presses the send button. Clearly both features influence each other. The send immediately feature should be disabled if the user is working off-line. However, this is not the case in Outlook. At present, Outlook still tries to send a message even though the user is working off-line. A potential cause for this problem could be that both features were implemented independently from each other. The problem only surfaces when both features are enabled. Consequently, unit testing will not detect this problem.

Feature interactions, like the example of Outlook, are very common in large software systems. As Zave observes, this type of problem potentially makes the composition of features incomplete, inconsistent, nondeterministic, hard to implement, etc. (see [10]). The method introduced in this paper aims to keep the composition complete, consistent, deterministic and implementable.

\subsection{Roles, actors and base components}

The modelling of an SPF as a base implementation composed of a set of selected features demands a more detailed modelling of a feature. Our approach does not assume how the inner workings of the base components are defined, only the assumption that some of them exist. The relationship between a feature and the base components is defined through a role-based approach.

Role modelling is used, because features typically affect more than one domain component simultaneously. In this perspective a feature can be viewed as a collection of base components playing roles of a feature. However, there is no simple one-to-one relationship between the roles of a feature and the roles 'played' by the base components. This simple relationship does not exist, because we want to define the features and their roles independent of the base components. As a consequence of this flexibility, there may be a base component playing more than one role of a feature. The opposite is also possible - one role of a feature can be played by several base components. To model this manyto-many mapping of roles and base components, our approach uses the concept of actors.

Note that with the term 'actor' a different concept is meant than is used in parallel object-oriented programming [11]. In the feature model, an actor is a first-class representation of a base component and the roles it plays for a single feature.

Figure 1 visualises the various feature model elements and their relationships. The base components visualised in the top part of Fig. 1 are entities, which cannot easily be decomposed into features and belong to the base SPF implementation. On the left side of Fig. 1 two features containing one and two roles are presented, visualising the fact that roles are part of a feature. At the centre there are four actors.

The top two actors consist of base components 1 and 2, both playing role 1 . The bottom two actors are base component 1 playing role 2 and base component 2 playing role 3 . At the bottom of Fig. 1 the derived components are situated. A derived component is a base component incorporating actors playing the roles of the selected features.

The concepts we discussed here form the basis of our composition approach, which will be elaborated on in Section 3. Before that, however, we provide an example.

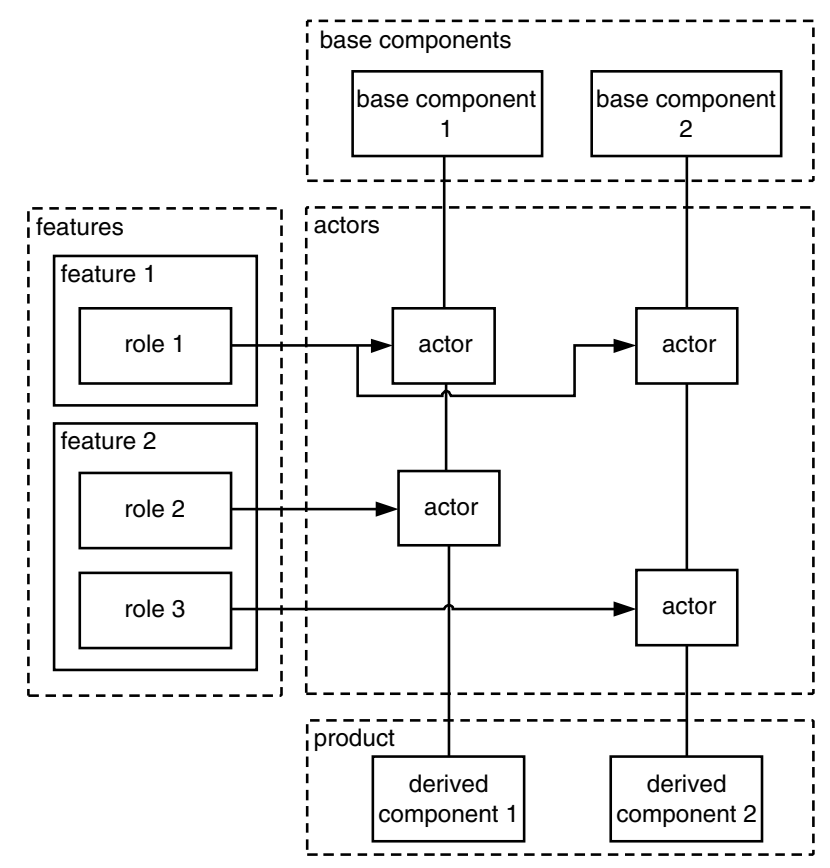

Fig. 1 Conceptual view of feature model 


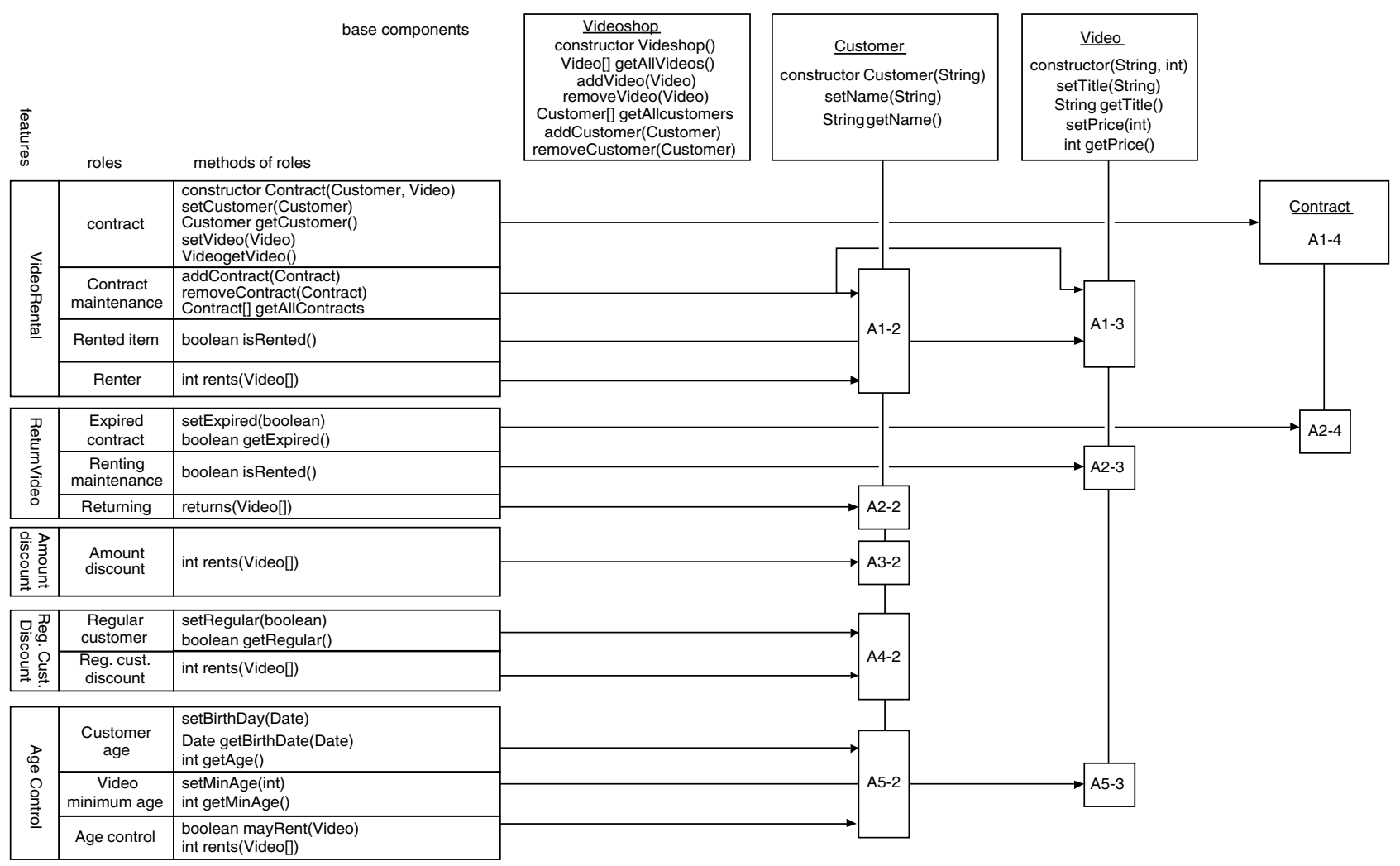

Fig. 2 Features, roles and actors of video shop renting system

\subsection{Case}

Throughout the rest of this paper a video renting administration system is used to illustrate various aspects of the feature model. A quick domain analysis provides the following domain components for the video-shop system: a VideoShop component, a Video component and a Customer component. These three domain components are the base components of this case. For the remaining part of the paper components are typeset in bold. Features are typeset underlined. The following features have been selected for this case:

- VideoRental: A Customer can rent a Video

- ReturnVideo: A Customer can return a Video that is rented

- AmountDiscount: A Customer receives a certain discount when renting more then one Video

- RegularCustomerDiscount: A regular Customer receives a certain discount when renting a Video

- AgeControl: Only a Customer above a certain age may rent a certain Video.

These features are selected because they illustrate the various issues of the feature model. The system should always contain the features VideoRental and ReturnVideo, for the system to have a minimal of functionality. Both features, however, will not be part of the base components because the specification of the features might change over time. The other features are optional. Some features are dependent on each other, e.g. all optional features depend on VideoRental. Also, some features will have feature interaction, for example AmountDiscount and RegularCustomerDiscount, both influence the amount of $\overline{\text { money a }}$ customer has to spend.

Figure 2 presents an overview of the video-shop case. The different features of the video-shop, e.g. VideoRental,

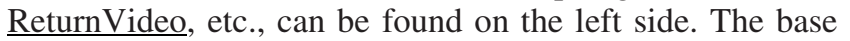
components (Videoshop, Customer, Video) are found on the top. The features consist of one or more roles, for example the VideoRental feature consists of the following roles (roles are in teletype): Contract, Contract Maintenance, RentedItem, Renter and Maintenance.

The first role, Contract of the VideoRental feature, introduces a new concept into the feature model. The Contract role introduces a new component in the composition, the Contract, which is not directly related to any existing base component. New concepts in the domain may be added by roles defining new components.

Because features are decomposed further into roles, the core of our composition method consists of mapping the defined roles onto the base components. The mapping of the different features and roles is visualised in Fig. 2. For each of the features the roles are presented and the functionality of the roles is visualised by displaying the signatures of the corresponding methods. Furthermore, the functionality of the base components is visualised.

When a role is mapped onto a base component an intermediate component is created (we refer to these intermediate components as actors); these are the little rectangles in the middle. Each actor has a unique name, for example the name of the Contract actor is A1-4. The first number indicates that the actor belongs to the first feature (i.e. VideoRental). The second number indicates the base component to which the role has been mapped. The Contract role has been mapped to a new 4th base component.

\section{Formalising the notion of features}

Composition of features such as described in Section 2 is far from trivial. Therefore, to be able to identify potential composition problems, the notion of features is formalised. In Section 4, we use this formal model to derive the properties of the composition operator. 
The feature model is formally defined in terms of sets. Mappings of elements (e.g. $A \rightarrow B$ ) of one set to elements of another set denote relationships between those elements.

In the model a method signature is denoted by an operationSignature, a unique identifier for the complete definition of the method itself without an implementation; for example, in Java this is the header of a method, including the method name, the list of parameters, and the type of the returned value. A set of such signatures is called an interface:

$$
\text { interface }=\left\{\text { operationSignature }_{i} \mid i \in \text { signatureSet }\right\}
$$

With this notation an interface is denoted as a set of operation signatures, named operationSignature ${ }_{1}$, operationSignature $_{2}$, etc., where signatureSet is the complete set of the available operation signatures.

A role is a set of interfaces and a one-to-one mapping of the operation signatures of the interfaces to implementations of these operation signatures. In imperative languages this implementation can be seen as a code block, i.e. the body of a method without the header.

A role can now be defined as:

role $=\{$

$\left\{\right.$ interface $_{k} \mid k \in$ interfaceSet $\}$,

\{operationSignature $_{k_{i}} \rightarrow$ implementation $_{k_{i}} \mid$

$k \in$ interfaceSet $\wedge i \in$ signatureSet $\left.\left._{k}\right\}\right\}$

The mapping describes that an operation signature is implemented by associating an implementation with the operation signature. A role is a partial implementation, mapped onto a component.

Separate roles in a feature are required to model the fact that one component can have multiple roles in the context of a feature. To do the mapping of a role onto a base component an intermediate form may be used. In a feature the implementations are mapped onto actors. An actor is a set of roles from a feature, mapped to a base component. An actor can be seen as an intermediate component.

A feature is a set of roles, a set of actors, and a many-tomany mapping from roles to actors, i.e.:

feature $=\left\{\left\{\right.\right.$ role $_{r} \mid r \in$ roleSet $\}$,

$\left\{\right.$ actor $_{o} \mid o \in$ actorSet $\}$,

$\left\{\right.$ role $_{i} \rightarrow$ actor $_{j} \mid i \in \operatorname{roleSet} \wedge j \in$ actorSet $\}$

A role may be mapped to more than one actor. Also, more than one role can be mapped to the same actor. One role can map to more than one actor, if the corresponding code is going to be used in more than one component. Although this will in general be considered a signal of bad design, it is not excluded in our model.

A software product family (SPF) consists of all features and all base components:

$\mathrm{SPF}=$

$\left\{\right.$ feature $_{f} \mid f \in$ featureSet $\} \cup$

$\left\{\right.$ baseComponent $_{o} \mid(o \in$ baseSet $\left.)\right\}$

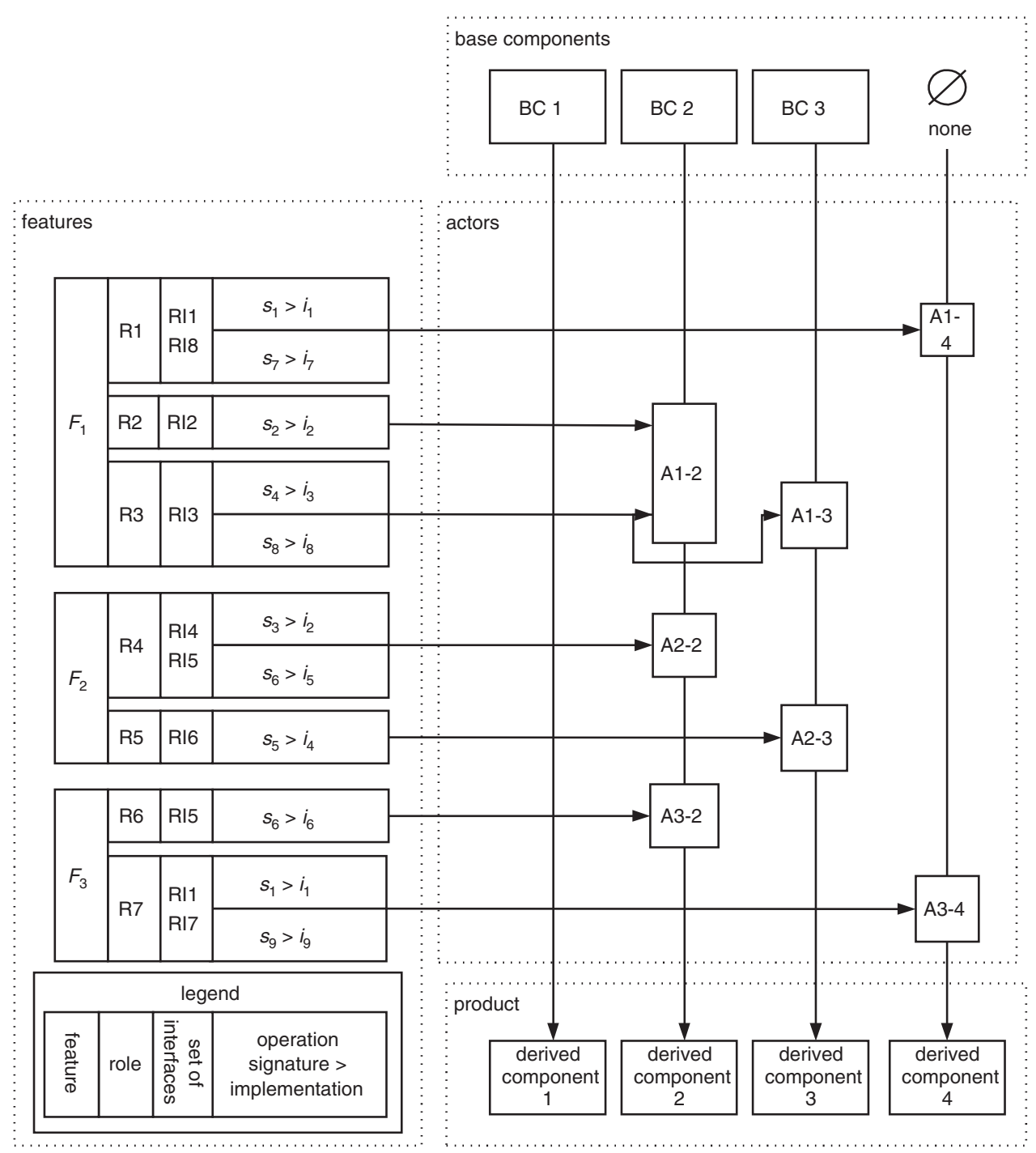

Fig. 3 Graphical representation of feature composition 
A specific product, derived from a SPF, consists of a selected number of features, a set of derived components and the mapping from the actors to the derived component implementations, which in turn are derived from the base components, i.e.:

product $=$

$\left\{\left\{\right.\right.$ feature $_{s} \mid s \in$ selectedFeatures $\subseteq$ featureSet $\}$, $\left\{\right.$ component $_{c} \mid(c \in$ compSet $\}$,

$\left\{\right.$ actor $_{i} \rightarrow$ component $_{i} \mid i \in$ actorSet $_{s} \wedge j \in$ compSet $\left.\}\right\}$

The set of derived components is derived from the set of base components, through the mapping of the actors to the base components. The component set is explicitly included, as there can be a need for base components which do not have roles mapped on them but are used by included features.

For example, this is the case for $\mathrm{BC} 1$ in Fig. 3. As a consequence of this definition, the set of derived components contains at least as many elements as the set of base components, i.e.

$$
\left\{\text { baseComponent }_{o}\right\} \subseteq\left\{\text { components }_{c}\right\}
$$

The transformation from a base component to a derived component is not formalised here. This transformation is the main issue in our approach and is investigated further in the following Sections.

Figure 3 illustrates our approach: methods are mapped onto the components, through the actors. Actors may introduce new components, which are independent of the defined base components.

These new components are dependent on an additional, initially empty, base component none.

Note that we have introduced three types of components: the base components, new components and derived components. The base components come from a domain model or are legacy components. The new components are components introduced by the roles of new features. The derived components are components generated for a specific derivation.

Our model currently does not model feature dependencies. The reason for this is that modelling dependencies complicates the feature model too much for our purposes. Therefore, the assumption has been made that the dependencies among the features are known and can be resolved before applying the composition operator.

Feature dependencies only influence the order in which the composition operator is applied. However, this does not affect how the composition operator should work. Consequently we do not explicitly model feature dependencies because they are not relevant for our purpose of examining the properties of the composition operator.

\section{Composition operator}

In this Section, the composition operator for the feature model is investigated. The composition operator in the feature model is used to compose features with base components. A feature, however, consists of one or more roles that map onto an actor. This Section investigates how an actor can be composed of roles and base components, what the associated composition problems are and how these problems may be solved.

\subsection{Introduction}

An actor consists of roles and base components. In the feature model, the derived components contain the functionality of the corresponding base components and actors of the selected features that are mapped to the base component. As mentioned earlier, feature dependencies

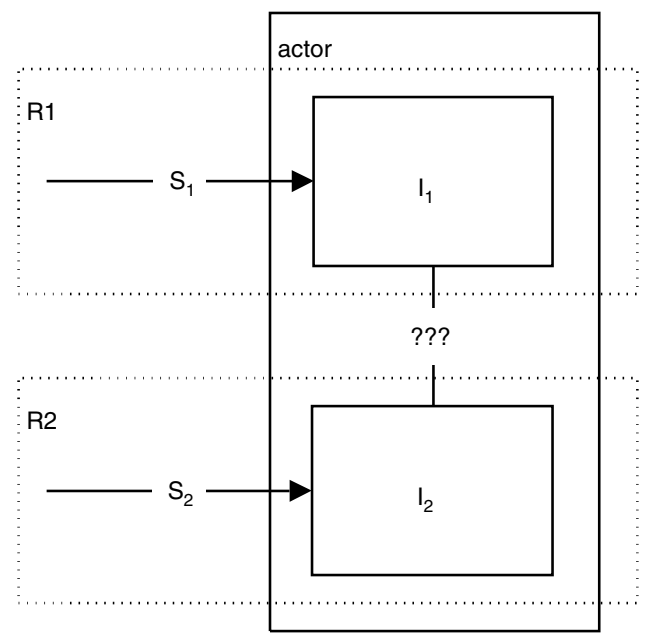

Fig. 4 Composition of an actor

complicate the composition process. Ideally the order in which features are mapped to base components would not affect the semantics of the derived components. However, because of the dependencies the order does matter (i.e. the composition operator is not commutative). Conceptually, the actors are accumulated on top of the base components (i.e. each actor is composed with the composition of all previous actors and the base components). Each actor combines various roles of a feature that are mapped to the same base component.

For example, Fig. 4 visualises the composition of two roles (R1 and R2). To simplify the composition problem, Fig. 4 does take into account that an actor can be composed with a base component or another actor. However, this has no consequences for the composition operator. Both roles (R1 and R2) consist of one operation, denoted by $S_{1}$ and $S_{2}$, and an implementation for this operation (i.e. $\mathrm{I}_{1}$ and $\mathrm{I}_{2}$ ). The actor that results from the composition of role $\mathrm{A}$ and $\mathrm{B}$ should contain the unified behaviour of roles A and B. Both implementations $\mathrm{A}$ and $\mathrm{B}$ are considered to be black boxes. The composition of the actor then becomes the problem of 'gluing' both implementations together, as denoted in Fig. 4 with the question marks.

\subsection{Analysing the composition of roles}

Both operation signature and implementation have an effect on the 'glue' that is needed to compose the implementations. By looking at the relationships between the operation signatures and the implementations, four different types of composition can be identified:

1. Signatures and implementations are all different. Figure 3 illustrates this: roles R2 (with $s_{2} \rightarrow i_{2}$ ) and R6 (with $s_{6} \rightarrow i_{6}$ ). R2 is mapped onto actor A1-2 and R6 is mapped onto actor A3-2. Both A1-2 and A3-2 are mapped onto the same base component BC2. An example in the video shop (Fig. 2) is the Renter and Returning roles.

This situation does not raise any problems because there is no interaction between the implementations.

2. The signatures are different and the implementations are equal. In Fig. 3 this is illustrated in roles R2 (with $s_{2} \rightarrow i_{2}$ ) and R4 (with $s_{3} \rightarrow i_{2}$ ). Role R2 is mapped onto actor A1-2 and R4 onto A2-2. Both A1-2 and A2-2 are mapped onto the same base component BC2. The video shop example does not contain this situation.

This situation does not present any problems either. It might signal bad design because different signatures can be 
implemented using the same implementation so the signatures might be considered equal instead of different.

3. Both the signatures and implementations are equal. This looks like copy-paste reuse and also a bad practice. In Fig. 3 this is illustrated in roles R1 (with $s_{1} \rightarrow i_{1}$ ) and R7 (again $\left.s_{1} \rightarrow i_{1}\right)$. R1 is mapped onto actor A1-4, R7 onto A3-4 and both actors are mapped onto the same base component $\mathrm{BC} 4$. The video shop example does not contain this situation. Although code fragments appear double in the resulting application there are no serious problems. Problems may arise, however, when maintenance is needed (code needs to be repaired in different places). A simple solution for this kind of problems is simply mapping the different code fragments to just one fragment.

4. The signatures are equal and the implementations are different. In Fig. 3 this is illustrated in roles R4 (with $s_{6} \rightarrow i_{5}$ ) and R6 (with $s_{6} \rightarrow i_{6}$ ) Role R4 is mapped onto actor A2-2, R6 onto actor A3-2 and both actors are mapped onto the same base component $\mathrm{BC} 2$. In the video shop case an example of this situation can be found with the operation rents in the roles Renter and AmountDiscount. This is a serious problem that requires further investigation. The remainder of this Section is devoted to this problem.

Of the four described combinations for composing the roles, only the last one is problematic. The combination of one signature with more than one implementation can be illustrated best with Lego building blocks. An operation signature is the header and the implementation the body of a method. Equal operation signatures therefore means that the headers are equal, and thus the parameter list and return type of the methods implementing the operation signature are equal - only the body is different.

A Lego brick can be seen as a shape representing the operation signature: the shape of the top of the brick illustrates the parameter list and the shape of the bottom of the brick illustrates the return type. The inside of the brick represents the implementation. Because in situation four, the signature is the same for both implementations, the Lego bricks have the same shape. This results in three ways to combine the implementations, as illustrated in Fig. 5:

A. No input parameters, no output. The operation signature of the implementations has no return type and no parameters. This is the easiest situation because the implementations may just be concatenated.

B. Input is equal to output. If the implementations have the same input type as the return type, the implementations may be piped together. However, this requires that the semantics of the input and output match.

C. Input and output are different. In this situation the implementations can neither be concatenated nor piped together. Some glue code may be needed to transform both implementations into one implementation.

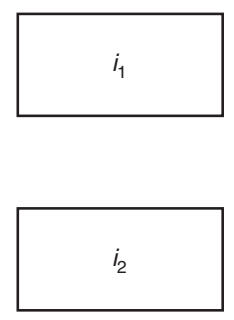

situation $\mathrm{A}$
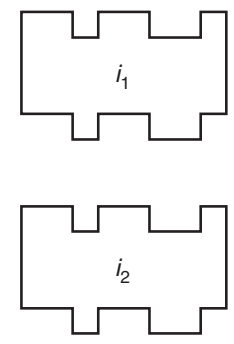

situation $B$
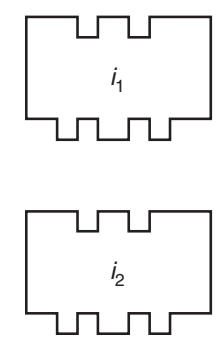

situation C
Fig. 5 Graphical representation of variations of having one signature with two implementations

192
In all cases a transformation is needed that combines two different implementations into one implementation for the same operation signature. In Lego terms this compares to building a new stone with the same shape (i.e. with the same input parameters and output parameter). Problems arise because of initialisation at the beginning of each of the two implementations, the output parameters of each of the implementations, and side-effects such as, for example, exception handling and results arising.

Any implementation of a feature composition will need to make specific design choices with respect to these transformations. Next we look at three alternatives for these transformations. In Section 5, we discuss a prototype algorithm we developed in Java where several design choices with respect to these transformations are made.

\subsection{Composing implementation blocks}

There are several ways of combining the two implementations with the same operation signatures, but there are three basic forms:

- Concatenation: The implementations can be concatenated: $i_{a} ; i_{b}$ or $i_{a} ; i_{b}$. Concatenating the implementations can only be done if the output of the first implementation can be used as input for the second. Thus, concatenation can only be used in situations A and B of Fig. 5. Even then, sideeffects such as exception handling may prevent successful concatenation.

- Skipping: One of the implementations can be skipped: $i_{a}$ or $i_{b}$. Skipping one of the implementations requires an additional criterion to be able to select which one of the implementations to skip.

- Implementation mixing: The implementations may be mixed (e.g. by superimposing [12], inheritance or delegation). Mixing the implementations, the last possibility of the three, requires knowledge of both the implementations $i_{a}$ and $i_{b}$. Suppose $i_{a}$ is in a feature $F_{a}$ and $i_{b}$ is in a feature $F_{b}$ and $F_{a}$ is dependent on the feature $F_{b}$. Then it is possible to use $i_{b}$ everywhere in the code of $i_{a}$, because they have equal operation signatures. At this point this is best illustrated by comparing this kind of mixing with using an original method of a superclass in the redefined method in the subclass by calling super in Java.

There are a number of issues with the different combination strategies described above:

- Scope of variables: Both implementations may have a common set of local variables with different semantics, which may raise some conflicts when both implementations are combined. A possible solution is to automatically rename conflicting local variables.

- Side-effects: Both implementations may have conflicting side-effects. For example, both implementations may throw an exception. The code of the second implementation may never be executed if the first implementation throws an exception. There are many subtle ways both implementations may conflict which need to be considered when combining the implementations.

It should be pointed out that other approaches (e.g. AspectJ $[13,14])$ exhibit the same sort of problems. In particular, AspectJ has become a complex language due to the fact that it tries to solve/work around these issues.

\subsection{Summary}

In this Section the formal model of the feature composition model has been used to examine where exactly this composition becomes problematic, namely when combining 
implementations with the same signature into one implementation. We have outlined three strategies, that may be combined, for doing so. However, there are a number of issues that prevent a universal solution to this problem. Any implementation of our feature composition model requires that these issues be addressed in some way. In the following Section we will outline the composition algorithm we used to implement the video shop prototype.

\section{Prototype implementation of feature model}

In this Section a prototype implementation of the feature model is presented. The implementation has been implemented in the object-oriented programming language Java. The prototype, the implementation choices and potential alternative solutions are presented. The Section ends with an overview of implementation issues and their solutions.

\subsection{Prototype}

The prototype that is presented in this Section is intended as a proof of concept. Consequently, a limitation of this is that the prototype is missing some features that would be available in a complete implementation. The composition operator as implemented only supports one variant of the implementation mixing composition solution (see Section 4.3). In addition, an important limitation is that automatic product derivation is not supported with a compiler. Instead, the transformation of the features and the base components into the derived components is done manually. However, it is possible to automate this in the future.

The main motivation for building the prototype was to demonstrate that the composition technique described earlier can be implemented in a mainstream programming language, such as Java. However, features are not a firstclass entity in Java. Consequently, the feature model entities have to be mapped to Java language constructs. Some of the design choices regarding this mapping are:

- Feature: A feature is a collection of roles in the feature model. Both features and roles do not have a representation in the Java language. However, Java supports the concept of a package that may be used to group various classes together. The prototype uses the package construct to group roles of the same feature together.

- Role: In the feature model a role is a collection of interfaces and some code blocks. A Java class also has interfaces and code blocks. Therefore a role is implemented as a Java class in our prototype.

- Base component: Similarly, base components are also implemented as Java classes.

- Actor: The goal of feature composition is to combine the base components and the roles in such a way that the result is has the composed behaviour of both. When a role is mapped to a base component an intermediate placeholder class (i.e. an actor) is created that inherits from the base component class.

- Derived component: Derived components are the result of the composition of selected features and base components. As stated before, actors combine base components and roles using inheritance. However, the derived components should incorporate all the composed behaviour of all the actors and base components. Since Java does not have multiple inheritance, the derived component cannot be constructed by letting them inherit from all the actors classes. To work around this problem, actors inherit from each other. Consequently, the last actor of a base component will have the required composed behaviour of a derived component. Therefore the derived component is an empty placeholder Java class, which inherits from the last actor defined on a base component. An example of this can be found in Fig. 6. A more in-depth description of the composition process and an algorithm for the composition in the Java language can be found in [15].

In Fig. 6 a UML example of the prototype for the feature model of Fig. 1 is presented. The packages of the prototype are visualised as grey boxes which can contain other packagers or classes. The classes are the white rectangles and inheritance is illustrated as an arrow from the subclass to the parent class.

\subsection{Potential issues for automatic composition}

This Section presents a description of the various implementation issues that arose during the implementation of the prototype. The found issues are believed to be general for implementing automatic feature composition based on the feature model. The main issues found during the implementation of the prototype are:

- the lack of a dependency model in the feature model

- an instantiation problem in the roles

- traceability of roles, features and actors.

The lack of a dependency model in the feature model is an issue an implementation has to deal with. The feature model assumes that a feature earlier introduced is not dependent on a later feature. The fact that this does not have to be the case in an implementation requires modelling of the dependencies at least at the implementation level. Dependencies also help to define the context of a feature in which a role is specified; hence it restricts the knowledge an implementer needs to implement a role. Experience has taught that there are four types of dependencies:

- Dependency between roles of the same feature: An example of dependencies among roles in the same feature can be found in the VideoRental feature of the video-shop case (see Fig. 2). One can only rent a Video if the concept of a Contract is introduced (this is in the Contract-role) and the necessary Contract operations in the Customer and Video (these are in the ContractMaintenance-role) are known.

- Dependency between roles of different features: Dependencies between roles of different features are the basis for feature dependencies. Feature dependencies in the feature model are the result of roles depending on roles or actors of a different feature. An example is the role ExpiredContract of ReturnVideo, which is dependent on the VideoRental feature, because of the needed concept of a Contract. This concept is introduced in the VideoRental feature by the Contract role.

- Dependency between a role and an actor: This dependency is different from a role depending on another role, because in this dependency a role is dependent on the composition of a role and a specific base component, which is an actor. An example is the Returning role of the Return-Video feature. The Contract that the Returning role uses should have the Contract role (from the VideoRental feature) and also the ExpiredContract role (from the ReturnVideo feature), to be able to expire a contract for this Customer.

- Dependency between a role and a composition of multiple actors. This dependency is a dependency between a role and a composition of roles and a base component. An example 


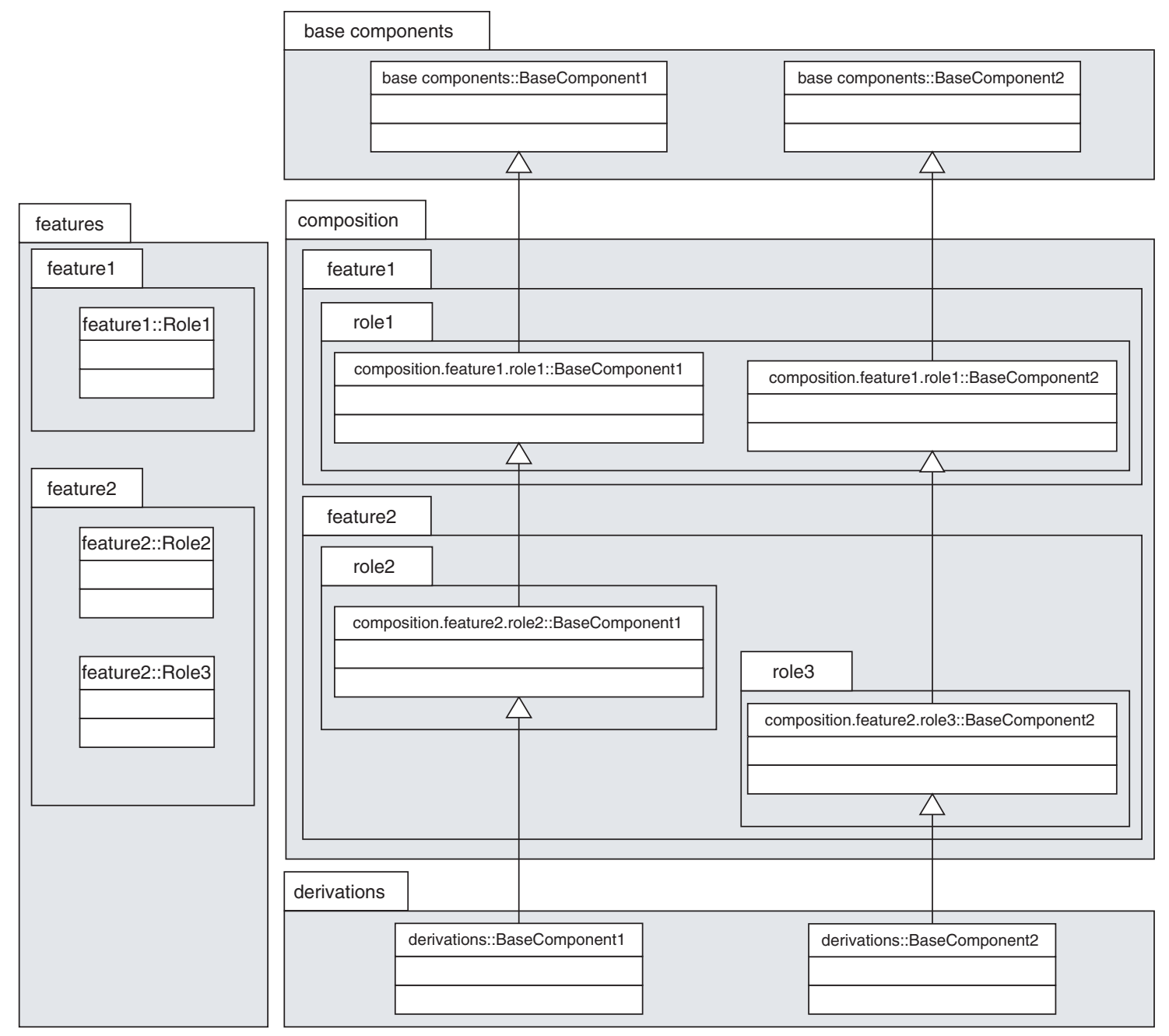

Fig. 6 UML diagram of prototype implementation of Fig. 1

of this dependency is in the Returning role of the ReturnVideo feature. The Video returned should contain the ContractMaintenance role and the RentingMaintenance role to be able to determine whether the Video is already rented and to add a new Contract to the Video if this is not the case.

The prototype implements the two role related dependencies with the help of the traditional Java dependency model (i.e. the use of the import statement). The two other dependencies are only partially realised. The recursive way actors are composed, makes the use of traditional Java dependencies between a role and an actor semantically different.

The second implementation issue is the instantiation problem. At the moment the roles are written it is not determined which class should be instantiated, because other features can be added or removed on the fly. Observe that the last actor of a component contains the complete composed behaviour for that component; this is due to the recursive composition behaviour of the actors. Each of the components has its own derived component, which should contain the complete composed behaviour for that component depending on the selected features.

The derived component therefore could inherit from the last defined actor for the component. If, during the derivation process, the derived component confirms to this inheritance and has a stable name, then it can be instantiated in the different roles.
Another implementation issue is the traceability of roles, features and actors, which is required for debugging the derived components. In the prototype the traceability of the different actors is accomplished by the first class representation of the actors. The name of the package in which the actor class is defined is determined by the feature and role names. The name of the actor class is equal to the name of the base component on which it is mapped, resulting in a complete reverse mapping from the derived components back to the roles, features and base components.

\subsection{Summary}

This Section presented a prototype implementation of the feature model. The composition process used in the implementation was motivated and explained. The concrete form of features, roles and actors in the implementation was presented. Implementation issues such as the lack of a dependency model in the feature model, traceability of roles, features and base components, and an instantiation problem were discussed.

\section{Related work}

This Section provides an overview of related work and their relationship with this paper. Separation of concerns, features, role modelling, and software product families and software architecture are the four areas of interest of which related work is examined. 


\subsection{Separation of concerns}

Separation of concerns is the appliance of the divide-andconquer paradigm on software design. By separating different concerns in separated entities the design becomes easier, but the 'gluing' of the pieces becomes harder.

Subject orientated programming (SOP) [16] uses the concept of different views on an entity. Each view has it own object hierarchy. Composition rules define how the different object hierarchies can be combined into a single unified object hierarchy. Our approach differs in the focus, which is on the collaboration aspect of feature related variability and not on functional hierarchical differences.

Aspect oriented programming (AOP) [13] uses the concept of aspects to capture functionality that is crosscut in normal object decomposition. So-called join points provide hooks to merge aspects with the objects. One of the implementations of AOP is AspectJ [14]; here the join points are the method activations. A conceptual model stating what aspects are is missing in AOP. The presented feature model in this paper can be used as a conceptual model for aspects, with the aspects implementing the composition of our feature model.

Multi-dimensional separation of concerns [17], as implemented in the HyperJ [18] approach, models different concerns in independent individual dimensions. Rules defining the relationships between the independent entities of the dimensions guide the necessary composition process for system generation. Our feature model can be viewed as a two-dimensional instance of a multidimensional separation of concerns model. The first dimension is the concern of the base components, the second the feature related variability dimension. The resulting matrix of actors is very similar to a composition expression in hyperslice programming. However, our model is different as it explicitly distinguishes features, and adds additional semantics (interface, roles) and notation (see Fig. 3). The feature model is not restricted to only these two dimensions of separation of concerns, because no restrictions on the dimensionality of the base components or features are defined.

The composition problem found in this paper (see Section 4 ) is universal for multi-dimensional separation of concerns, because each concern model will only describe a part of the behaviour of an entity. However, each concern model needs to overlap/relate to other concern models, otherwise a composed view of an entity is not possible. Multidimensional separation of concern therefore has the inherent problem that an operation of an entity could have multiple behaviours that should be combined, resulting in a composition problem.

\subsection{Features}

A more global view of how features can bridge the gap between the problem and solution domain is presented in [9]. In their view features are composed out of requirements fragments and realised in one or more design fragments, which make up the complete design. In this perspective, features can be seen as an example of early aspects [6] - a crosscutting concern during the requirement engineering and architecture design phase. This paper presents a more detailed description of how the design fragments, i.e. aspects, making up the feature can be modelled and composed for making the complete design.

Our approach is not unique in trying to model features in the solution domain. The feature-oriented domain analysis (FODA) [3] method is a method for identifying features during domain analysis. FODA uses the representation of feature trees to visualise the variability and dependencies of features. Later, the feature-oriented reuse method (FORM) [4], which is a superset of FODA, was developed to prescribe how the FODA feature model could be used to develop domain architectures and components for reuse. The main difference between our approach and FORM is the traceability of features at the design and implementation level, which is not the case with FORM. This traceability is lost during the FORM application-engineering phase.

Prehofer [19, 20], uses feature oriented programming (FOP) to compose features into objects. FOP is an extension of the object-oriented programming paradigm. It uses separate entities called lifters to model feature interaction and separates core functionality from feature functionality. Our approach differs in two ways; the first is that a first class entity (the actor) for the composed behaviour is present, enabling the definition of a feature based on the composed behaviour of two other features. The second difference is that a feature is not one static class but consists of different roles being mapped onto different domain components.

Zave [10] discusses a distributed feature composition technique (DFC) for telecommunication services. She uses a pipe \& filter style architecture, with the features being components, switches and routers connecting the components with connectors to form a chain of components through which data can move. Components can be added and removed by the switch, thereby changing the current feature set. The main difference with our approach is the fact that we do not require a particular architectural style to be used and features do not have to be contained in a single component.

The relationship between features and SPFs is also mentioned in [5], which proposes a feature-driven aspectoriented product-line CBSE. The main idea is to use a feature-driven analysis and design method like FeatuRSEB [21] to develop a feature model. One or more aspectorientated implementation techniques [22] can then be used to implement features in separate code fragments, which in turn can be composed based on the selected features for a given composition. The global idea is the same as the approach in this paper; however, the focus of this paper is more on the composition of features, whereas [5] is more a global modelling view.

\subsection{Role modelling}

The idea of role modelling has also been studied by other authors. The OOram method [23] uses role models based on collaborations of different roles. Two or more role models can be synthesised to form a new and more complex role model. The general role modelling ideas presented in OOram are used at various abstraction levels. The main difference with our approach is in the synthesis or composition of two role models. In OOram this is only done at a structural level; that is, only the structural requirements are validated - compositional problems have to be solved by the developer him/herself.

Role models can also be integrated into object-oriented frameworks [24]. The main focus of the role model used in this approach is on the interface part. The notation proposed in [24] could be used to denote the relationships between the roles of the features in our model. The main difference is that a coupling between an interface and an implementation of a role is not made in their model something that our feature model does. The composition problem we have identified is therefore not relevant in their approach, because the composition problem finds its roots in a coupling between an interface and an implementation. 
Fowler [25] defines design patterns that can be used to implement roles in an object-oriented language. The main concept of the patterns is that the objects are dealing with a single object that has multiple changeable types. An object is therefore aware of the multiple typing of the other objects and has to act on this, which in the feature model does not have to be the case, because we want to be able to develop unrelated features independently.

The use of mixins [26] - abstract subclasses representing a mechanism for specifying classes that will eventually inherit form a super class - is another approach to compose collaborations. The difference with our approach lies in the mapping of the roles of the collaborations to the objects. These can only be mapped to a single domain object, and multiple roles cannot be mapped to the same domain object - something our feature model can.

\subsection{Software product families and software architecture}

The software product family (SPF) [1] is an approach to develop software, not on an application base but on the basis of a family of related applications. The commonalities between the individual products (applications) can be used to create so-called common assets, which are reusable components that can be customised for the individual products. The field of variability management [27] of SPFs mainly concerns how the differences between the products can be managed. This paper presents a method about how the common assets can be customised for a specific product based on a selection of features and is therefore a form of variability management.

In the context of SPF, [28] also use features to customise the common assets to derive a product based on a selection of features. They use packages as features and the merging of source trees to accomplish feature composition. The merging of the source trees takes place at so-called variation points. A variation point in their approach is a simple switch statement, defining the different variations on the code level. A problem with this approach is the definition of the variation points. The variation points have to be programmed out manually in the form of switches, and this mixes the feature related code with the common asset code. This reduces the traceability and the reuse capabilities of the feature related code, because of the lack of first class representation, which is present in our approach.

The software architecture (SA) [29] of each derived product is a variant of the SPF architecture. The feature model incorporates the components of the SPF architecture through the use of the base components. The feature model can therefore modify the architecture of a product by adapting the existing base components by mapping new roles of features on it and introducing new connectors and components resulting from features.

\section{Conclusions and future work}

In this paper, we have investigated the potential of using the early aspect of features in the solution domain of software product families (SPFs). Our main focus was on the design and implementation level. Starting at the design level a feature model was presented, modelling features. The model showed how features could be modelled as a collection of roles, thereby relating for the first time a feature model to a role model. The roles in turn can be played by different basecomponents, resulting in actors. At the implementation level a way to implement the model is outlined. The model and the outlined implementation strategy are illustrated and validated with a prototype implementation.
With the help of a formalised version of the model a compositional problem is identified. The composition of two roles in an actor becomes problematic when both roles have different implementations for the same method. To solve the composition problem there are three potential solutions: skipping, concatenation and mixing. One or more of the three solution forms can be used to solve composition problems.

By predefining how the composition is done and which composition solution to use in the case of a composition problem, we can keep the composition of our feature model complete, consistent, deterministic and implementable. This facilitates the automatic derivation of products in an SPF based on a selection of features.

Remaining open issues that we intend to address in future work are:

- Automatic composition support: An open issue of the prototype is the absence of a compiler that supports the composition process. At the moment the necessary composition steps still have to be done manually, which makes the application of the composition process a very time-consuming and error-prone one. However, we have already defined an algorithm for the composition process, which can easily be programmed out, automating the composition process.

- Scalability of the feature model: Scalability of the feature composition model is one of the main aspects that demand additional validation. Although the feature composition model was designed for use in SPFs, it has not yet been demonstrated whether the model can be scaled up to this level of scale.

- Lack of a dependency model: The feature model does not include a dependency model. The combinations of features that are possible for product derivation are directly related to the feature and roles dependencies. So, it is important to extend the feature model with a dependency model. The first steps have already been taken in Section 5.2, where four dependency relation types are already identified.

- Validation of the feature model: A correct and complete validation of the feature model is difficult to accomplish. Cases can be used to validate the feature model, as does the video-shop case in this paper, for example.

These open issues form the basis for further work. We would like to investigate how automatic composition support can help with the scalability of the feature model. For decent automatic composition support the feature model should be extended to include a dependency model. Additional research is planned with an additional case helping us to investigate the scalability of the feature model and providing additional validation of our approach.

\section{References}

1 Bosch, J.: 'Design \& use of software architectures - adopting and evolving a product-line approach' (Addison-Wesley, Harlow, 2000)

2 Bosch, J.: 'Maturity and evolution in software product lines: approaches, artefacts and organization'. Proc. 2nd Software Product Line Conf. (SPLC2), San Diego, August 2002, pp. 257-271

3 Kang, K.C., Cohen, S.G., Hess, J.A., Novak, W.E., and Peterson, A.S. 'Feature oriented domain analysis (FODA) feasibility study'. Technical Report CMU/SEI-90-TR-21, Software Engineering Institute, Carnegie Mellon University, Pittsburgh, PA

4 Kang, K.C.: 'FORM: a feature-oriented reuse method with domainspecific architectures', Ann. Softw. Eng., 1997, 5, pp. 354-355

5 Griss, M.L.: 'Implementing product-line features by composing component aspects'. Proc. 1st Int. Software Product Line Conf., Denver CO, August 2000, pp. 271-288

6 Rashid, A., Sawyer, P., Moreira, A., and Araujo, J.: 'Early aspects: a model for aspect-oriented requirements engineering'. Proc. IEEE Joint Int. Conf. on Requirements Engineering, Essen, Germany, 9-13 September, 2002, pp. 199-202 
7 Gibson, P.: 'Feature requirements models: understanding interactions'. Presented at IEEE 4th Int. Workshop on Feature Interactions in Networks and Distributed Systems (FIW), Montreal, Canada, June 1997

8 Griss, M.L.: 'Implementing product-line features with component reuse', Lect. Notes Comput. Sci., 2000, 1844, pp. 137-152

9 Turner, C.R., Fuggetta, A., Lavazza, L., and Wolf, A.L. 'A conceptual basis for feature engineering', J. Syst. Softw., 1999, 49 (1), pp. 3-15

10 Zave, P.: 'Feature-oriented description, formal methods, and DFC' Proc. FIRE-works Workshop on Language Constructs for Describing Features, Glasgow, 15-16 May 2000, pp. 11-26

11 Agha, O.A.: 'Actors: a model of concurrent computation in distributed systems' (MIT Press, Cambridge, MA, 1986)

12 Bosch, J.: 'Superimposition: a component adaptation technique', Inf. Softw. Technol., 1999, 41, pp. 257-273

13 Kiczalez, G., Lamping, J., Mendhekar, A., Maeda, C., Lopes, C. Loingtier, J-M., and Irwin, J.: 'Aspect oriented programming'. Proc. 11th European Conf. on Object-oriented Programming (ECOOP), Jyväskylä, Finland, 9-13 June 1997, pp. 220-242

14 Kiczales, G., Hilsdale, E., Hugunin, J., Kersten, M., Palm, J., and Griswold, W.G.: 'An overview of AspectJ', Lect. Notes Comput. Sci. 2001, 2072, pp. $327-353$

15 Jansen, A.G.J.: 'Feature based composition'. Master Thesis computer science, University of Groningen

16 Harrison, W., and Ossher, H.: 'Subject-oriented programming a critique of pure objects'. Proc. 1993 Conf. on Object-Oriented Programming Systems, Languages, and Applications (OOPSLA), Washington, DC, 26 September-1 October 1993, pp. 411-428

17 Tarr, P., Ossher, H., and Harrison, W.: ' $N$ degrees of separation: multidimensional separation of concerns'. Presented at Int. Conf. on Software Engineering (ICSE), Los Angeles, CA, 16-22 May 1999, pp. 419-443

18 Ossher, $\mathrm{H}$, and Tarr, P. 'Multi-dimensional separation of concern and the hyperspace approach', in Akcit, M. (Ed.): 'Software architectures and component technology' (Kluwer, Boston, 2001), Chapter 10

19 Prehofer, C.: 'Feature-oriented programming: a fresh look at objects', Lect. Notes Comput. Sci. 1241

20 Prehofer, C.: 'An object-oriented approach to feature interaction'. Presented at 4th IEEE Workshop on Feature Interactions in Telecommunications Networks and Distributed Systems, Montreal, Canada, 17-19 June 1997

21 Griss, M.L., Favaroe, J., and d'Alessandro, M.: 'Integrating feature modeling with the RSEB'. Proc. 5th Int. Conf. on Software Reuse, Los Alamitos, CA, USA, 1998, pp. 76-85

22 Czarnecki, K., and Eisenecker, U.W.: 'Generative programming. methods, tools, and applications' (Addison-Wesley, Boston, MA, 2000)

23 Reenskaug, T.: 'Working with objects - The OOram software engineering method' (Manning, Greenwich, CT, 1996)

24 Riehle, D., and Gross, T.: 'Role model based framework design and integration'. Proc. Conf. on Object-Oriented Programming Systems, Languages, and Applications (OOPSLA), Vancouver, BC, 18-22 October 1998, pp. 117-133

25 Fowler, M.: 'Dealing with roles'. Presented at 4th Annual Conf. on Pattern Languages of programs'. Mantiello, Ullinios, USA, 2-7 September 1997

26 Smaragdakis, Y., and Batory, D.: 'Implementing layered designs with mixin layers'. Proc. 12th Eur. Conf. on Object-Oriented Programming (ECOOP), Brussels, July 1998, pp. 550-570

27 van Gurp, J., Bosch, J., and Svahnberg, M.: 'On the notion of variability in software product lines'. Proc. Working IEEE/IFIP Conf. on Software Architecture (WICSA), Amsterdam, 28-31 August 2001, pp. 45-54

28 van Deursen, A., de Jonge, M., and Kuipers, T.: 'Feature-based product line instantiation using source-level packages'. Proc. 2nd Software Product Line Conference (SPLC2), San Diego, CA, 19-22 August 2002, pp. 217-234

29 Bass, L., Clements, P., and Kazman, R.: 'Software architecture in practice' (Addison-Wesley, Reading, MA, 1998) 
Copyright of IEE Proceedings -- Software is the property of IEE and its content may not be copied or emailed to multiple sites or posted to a listserv without the copyright holder's express written permission. However, users may print, download, or email articles for individual use. 